\title{
Verifying Results of the IBM Qiskit Quantum Circuit Compilation Flow
}

\author{
Lukas Burgholzer* \\ Rudy Raymond ${ }^{\dagger}$ \\ Robert Wille*‡ \\ *Institute for Integrated Circuits, Johannes Kepler University Linz, Austria \\ †IBM Research - Tokyo, Japan \\ ${ }^{\ddagger}$ Software Competence Center Hagenberg GmbH (SCCH), Hagenberg, Austria \\ lukas.burgholzer@jku.atrrudyhar@jp.ibm.com robert.wille@jku.at \\ https://iic.jku.at/eda/research/quantum/
}

\begin{abstract}
Realizing a conceptual quantum algorithm on an actual physical device necessitates the algorithm's quantum circuit description to undergo certain transformations in order to adhere to all constraints imposed by the hardware. In this regard, the individual high-level circuit components are first synthesized to the supported low-level gate-set of the quantum computer, before being mapped to the target's architecture-utilizing several optimizations in order to improve the compilation result. Specialized tools for this complex task exist, e.g., IBM's Qiskit, Google's Cirq, Microsoft's QDK, or Rigetti's Forest. However, to date, the circuits resulting from these tools are hardly verified, which is mainly due to the immense complexity of checking if two quantum circuits indeed realize the same functionality. In this paper, we propose an efficient scheme for quantum circuit equivalence checking - specialized for verifying results of the IBM Qiskit quantum circuit compilation flow. To this end, we combine characteristics unique to quantum computing, e.g., its inherent reversibility, and certain knowledge about the compilation flow into a dedicated equivalence checking strategy. Experimental evaluations confirm that the proposed scheme allows to verify even large circuit instances with tens of thousands of operations within seconds or even less, whereas state-of-the-art techniques frequently time-out or require substantially more runtime. A corresponding open source implementation of the proposed method is publicly available at https://github.com/iic-jku/qcec
\end{abstract}

\section{INTRODUCTION}

Quantum computing has gained considerable momentum over the past years, as actual quantum computers are reaching feasibility and more and more algorithms for potential applications are discovered. Similar to the conventional realm, a conceptual quantum algorithm needs to be compiled to a representation that conforms to all restrictions imposed by the device it shall be executed on. To this end, fast-evolving compilation flows such as those of IBM's Qiskit [1], Google's Cirq [2], Microsoft's QDK [3], or Rigetti's Forest [4] are available.

Naturally, it is of utmost importance that the results of such compilation flows are correct, i.e., that the compiled quantum circuit still realizes the originally intended functionality. This motivates the development of methods for verification or, more precisely, equivalence checking. Conceptually, equivalence checking is simple, since each quantum circuit $G$ realizes a unitary transformation $U$ and comparing two quantum circuits $G$ and $G^{\prime}$ boils down to comparing the corresponding unitary matrices $U$ and $U^{\prime}$, respectively. However, since these matrices $U$ and $U^{\prime}$ are exponentially large, many existing approaches to this problem (e.g., [5]-[9]) remain unsatisfactory and can hardly be employed on a large scale.

Recently, a promising solution to address this problem has been proposed in [10]. There, the use of dedicated data-structures, in particular decision diagrams [9], [11], [12], has been proposed which allow for a non-exponential representation of matrices in some (albeit not all) cases. To further improve upon that, it is exploited that $G^{\prime-1} \cdot G=\mathbb{I}$ holds if two circuits $G$ and $G^{\prime}$ are indeed equivalent, i.e., the cascade composed of one circuit with the inverse of the other should eventually yield the identity function. Because of that, checking whether $G$ and $G^{\prime}$ are in fact equivalent can be conducted by starting with the identity matrix (which can be represented in linear rather than exponential space) and, then, applying operations of $G$ and $G^{\prime}$ in a particular order until all operations have been applied. If all those operations can be applied so that the respective intermediate computations remain as close to the identity as possible, substantial improvements can be achieved [10]. However, how to determine the "perfect" order of applications, i.e., whether to apply operations from $G$ or from $G^{\prime}$ in order to keep the respective intermediate representation close to the identity, remains an open problem 1

In this paper, we address this problem. We show that, by utilizing knowledge about the compilation flow, a verification methodology can be obtained which keeps the respectively occurring intermediate representations close to the identity in an almost perfect fashion. By this, the exponential complexity is frequently reduced to a linear or close-to-linear complexity - substantially reducing the runtime of the verification process. In order to showcase the possible improvements, we consider the compilation flow as it is currently conducted by IBM's Qiskit ${ }^{2}$ Experimental evaluations show that, using

\footnotetext{
${ }^{1}$ This is discussed and illustrated in more detail later in Section III

${ }^{2}$ However, the methods proposed in this work can be tailored to any other compilation flow as well.
} 
the proposed method, circuits composed of tens of thousands of operations can be verified within seconds or even less, whereas state-of-the-art techniques frequently time-out or require substantially more runtime. A corresponding open source implementation of the proposed method is publicly available at https://github.com/iic-jku/qcec.

The rest of this work is structured as follows: In Section II. we review the necessary basics of quantum circuits and the IBM Q systems needed to keep this work self-contained. Then, Section III reviews the compilation flow as it is conducted by IBM's Qiskit and elaborates on verifying results of this flow. Based on that, Section IV illustrates how all different aspects are exploited and consequently orchestrated to form a dedicated verification strategy. The strategy's performance is then evaluated in Section V, before Section VI concludes the paper.

\section{QuAntum Computing And The IBM Q Systems}

In this section, we briefly review the concepts of quantum computing [13] and introduce the notation used in this work. Besides that, we also review the considered platform, i.e., IBM Q systems [14]. For more detailed information, we refer the interested reader to the provided references.

\section{A. Quantum Circuits}

In quantum computing, the main computational unit is the qubit. In contrast to classical bits, a single qubit $q$ can be in an arbitrary superposition of the basis states $|0\rangle$ and $|1\rangle$, i.e.,

$$
|q\rangle=\alpha_{0}|0\rangle+\alpha_{1}|1\rangle
$$

with $\alpha_{0}, \alpha_{1} \in \mathbb{C}$ and $\left|\alpha_{0}\right|^{2}+\left|\alpha_{1}\right|^{2}=1$. An $n$-qubit quantum system can be in an arbitrary superposition of the $2^{n}$ basis states

$$
\left|b_{n-1}\right\rangle \otimes \cdots \otimes\left|b_{0}\right\rangle=\left|b_{n-1} \ldots b_{0}\right\rangle=\left|\sum_{i=0}^{n-1} b_{i} 2^{i}\right\rangle
$$

with $b_{i} \in\{0,1\}$, i.e.,

$$
|q\rangle_{n}=\sum_{i=0}^{2^{n}-1} \alpha_{i}|i\rangle \text { with } \alpha_{i} \in \mathbb{C} \text { and } \sum_{i=0}^{2^{n}-1}\left|\alpha_{i}\right|^{2}=1 .
$$

In the circuit model of quantum computation, qubits are represented by wires and are manipulated by quantum operations (quantum gates). Specifically, a quantum circuit $G$ with $m$ gates, operating on $n$ qubits, is denoted by $G=g_{0} \ldots g_{m-1}$, where each $g_{i}$ represents a quantum gate acting on (a subset of) $n$ qubits. This is usually visualized through quantum circuit diagrams, where the qubit wires are drawn as horizontal lines, gates are drawn using a variety of symbols, and progression of time is assumed to happen from left to right.

Example 1. An example of a quantum circuit $G$ with 16 gates acting on three qubits is shown in Fig. 1a. This sequence of operations describes a small instance of the famous Grover search algorithm [15]. The small boxes with identifiers correspond to operations applied to single qubits such as $X$ gates (the quantum analogue to the NOT gate) and H(adamard) gates (which can be used to set a qubit into superposition). Moreover, there are multiple-controlled $X$ operations, where an $X$ operation is only applied to a target qubit (denoted by $\oplus$ ) if all of its control qubits (denoted by $\bullet$ ) are in state $|1\rangle$. In case there is only one control qubit, such a gate is also called CNOT or controlled-NOT, while in case of two control qubits it is also called a Toffoli gate.

Initially, quantum algorithms are described in a way which is agnostic of the device they are planned to be executed on. However, physical devices today only support very low-level quantum operations. In this work, we focus on devices provided by IBM, which are briefly reviewed next.

\section{B. The IBM Q Systems}

In 2016, IBM launched the IBM Quantum Experience cloud platform which, for the first time, provides public access to a quantum computer. Today, eight devices with either one, five, or 15 qubits are freely available for development. All quantum computers developed at IBM provide the same limited gate-set consisting of arbitrary single-qubit gates $U$ and the two-qubit CNOT operation 3 Although seemingly small, this already constitutes a universal gate-set, i.e., every possible quantum computation can be realized using only gates from this set [13]. In addition, the IBM Q systems (or in general quantum computers based on superconducting qubits) feature a severely limited connectivity of their qubits. This is usually described by a coupling graph, where the graph's nodes represent the qubits and an edge between two nodes indicates that a CNOT operation may be applied to those qubits 4

Example 2. The coupling graph of the IBM $Q$ London system is shown in Fig. Ic It consists of five (physical) qubits $Q_{i}$ and a CNOT operation may only be applied to the qubits $\left(Q_{0}, Q_{1}\right),\left(Q_{1}, Q_{2}\right),\left(Q_{1}, Q_{3}\right)$, or $\left(Q_{3}, Q_{4}\right)$.

A device's physical qubits are inherently affected by noiseleading to rather short coherence times and limited fidelity of the individual operations. Until a certain threshold concerning the number of available qubits is reached, error correction is not yet an option. Throughout this work, we will refer to the physical qubits of a device using upper case $Q_{i}$ and denote the logical qubits of a quantum algorithm with lower case $q_{i}$. Additionally, $Q_{i}: q_{j}$ denotes that the logical qubit $q_{j}$ is assigned to the physical qubit $Q_{i}$.

\footnotetext{
${ }^{3}$ Specifically, $U=U_{3}(\theta, \phi, \lambda)$ with $0 \leq \theta \leq \pi$ and $0 \leq \phi<2 \pi$ are supported. Special cases are $U_{2}(\phi, \lambda)=U_{3}\left(\frac{\pi}{2}, \phi, \lambda\right)$ and $U_{1}(\lambda)=U_{3}(0,0, \lambda)$.

${ }^{4}$ Nowadays, these edges are typically undirected, i.e., it does not matter which qubit acts as control or target-whereas past architectures actually prescribed the direction.
} 


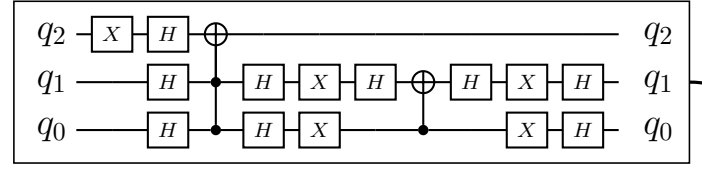

(a) Original circuit $G$

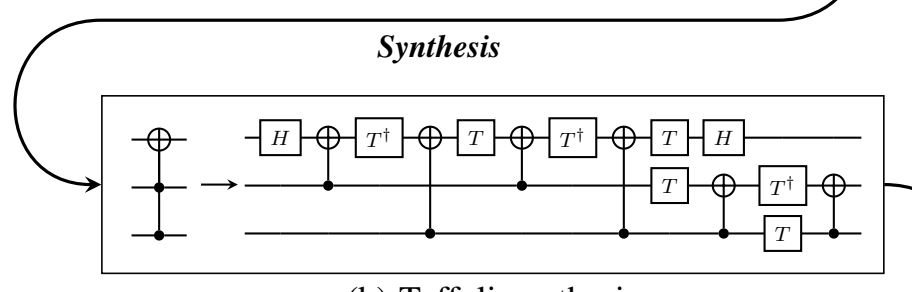

(b) Toffoli synthesis

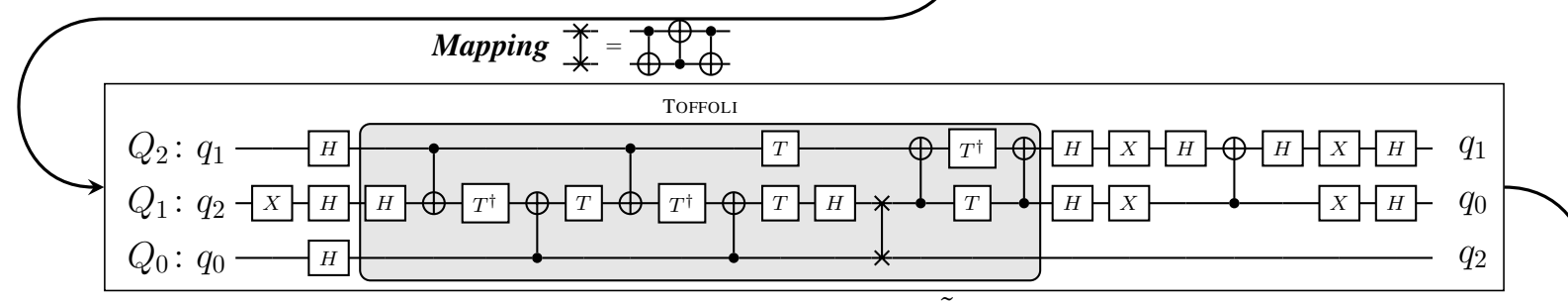

(d) Mapped circuit $\tilde{G}$

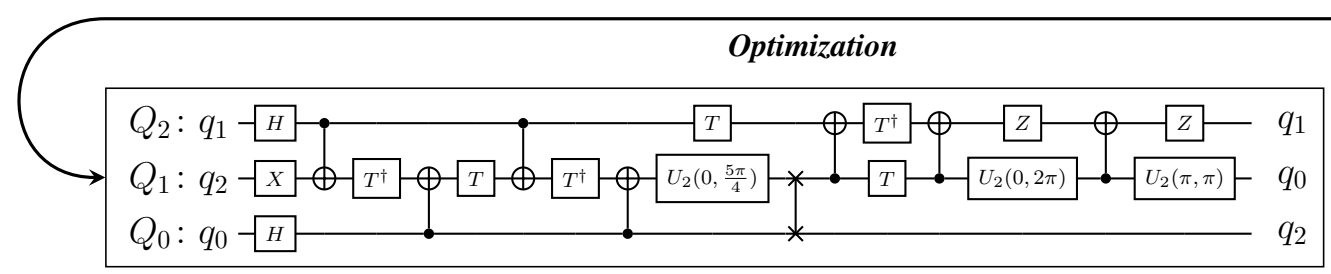

(e) Optimized circuit $G^{\prime}$

Figure 1: Exemplary illustration of the IBM compilation flow

\section{Motivation And Considered Problem}

In order to execute a conceptual quantum algorithm on an actual device such as provided by the IBM Q systems, several transformations have to be applied to the original circuit in order to conform to all the restrictions imposed by the targeted device. This transformation process is frequently called compilation ${ }^{5}$ due to its similarity to a classical compiler transforming high-level code into a (machine-executable) assembly. Many specialized tools for this complex task exist, e.g., IBM's Qiskit [1], Google's Cirq [2], Microsoft's QDK [3], or Rigetti's Forest [4]. However, to date, the circuits resulting from these tools are hardly verified, i.e., it is hardly checked whether the resulting quantum circuit description still realizes the same functionality as the originally given circuit. In the following, we review this compilation flow using IBM's Qiskit compilation flow as a representative. Afterwards, we elaborate on the problem of verifying results of this flow, which will eventually motivate this work.

\footnotetext{
${ }^{5}$ Other terms (interchangeably) referring or relating to (steps of) this process are synthesis, mapping, qubit allocation, qubit routing, or transpilation. In this work, we consider the complete process as compilation-split into the three main tasks synthesis, mapping, and optimization.
}

\section{A. The IBM Qiskit Compilation Flow}

Compilation of quantum circuits addresses the three kinds of restrictions which limit the usability of a quantum computer and have been reviewed above: The first two, i.e., the limited gate-set and connectivity, constitute hard constraintsa computation not conforming to these restrictions may not be executed on the device. In contrast, the short coherence time and limited gate fidelity represent a soft constraint-a quantum circuit may be executed on a device, but it is not guaranteed to produce meaningful results if the circuit, e.g., is too large for the state to stay coherent. The Qiskit compilation flow is structured as a collection of individual passes, each of which is responsible for dealing with a certain constraint (or an aspect thereof). Just as traditional compilers, there are different optimization levels offering a trade-off between compilation runtime and quality of the compilation result. More precisely:

First, the gates of the original quantum circuit are synthesized to the gate-set supported by the targeted device. Most importantly, since devices typically only support up to two-qubit gates, any gate acting on more than two qubits is broken down into "elementary" gates. This process may require the use of additional ancillary qubits for realizing the desired 
operation. In this regard, Qiskit provides several modes, e.g., for the decomposition of multi-controlled gates-offering a trade-off between circuit size and number of required ancillary qubits [16]-[18].

Example 3. Consider again the circuit $G$ from Ex. 1 as shown in Fig. Ia If this circuit shall be executed on an IBM $Q$ system, the Toffoli gate (the two-controlled NOT) first has to be realized using only arbitrary single-qubit gates and CNOTs. One possible synthesized version is shown in Fig. 1b. It takes six CNOTs, nine single qubit gates, and no additional ancillaries to realize the desired gate.

Now, the circuit just contains elementary gates supported by the device, but it may not yet conform to the device's limited connectivity. Thus, the quantum circuit is mapped to the target architecture, i.e., a mapping between the circuit's logical and the device's physical qubits is established. Qiskit provides several heuristics for determining an initial mapping-from a trivial one-to-one mapping $\left(Q_{i}: q_{i}\right)$ to explicitly considering calibration data and picking the most reliable set of qubits for the computation [19]. However, in most cases, it is not possible to globally define a mapping which conforms to all connectivity limitations. As a consequence, the logical-to-physical qubit mapping usually is changed dynamically throughout the circuit. Typically, this is accomplished by inserting $S W A P$ gates into the circuit-effectively allowing to change the mapping of logical qubits to physical qubits so that all operations can be executed while, at the same time, all connectivity constraints are satisfied. To this end, Qiskit per default uses a very fast, stochastic solution (based on Bravyi's algorithm). Several other approaches have been proposed for tackling this immensely complex task $[7],[19]-[25]$ and some of them have even been integrated into Qiskit.

Example 4. Consider again the circuit $G$ from Ex. 1 and assume that the Toffoli gate has been synthesized as shown in Fig. $1 b$ Further, assume that the circuit is to be executed on the IBM Q London architecture shown in Fig. $1 c$ Then, Fig. $1 d$ shows one possible circuit $\tilde{G}$ resulting from this mapping process. The physical qubits $Q_{0}, Q_{1}$, and $Q_{2}$ were chosen and initially assigned logical qubits $q_{0}, q_{2}$, and $q_{1}$, respectively. Just one SWAP operation applied to $Q_{0}$ and $Q_{1}$ (indicated by $\times)$ was added in the middle of the circuit in order to conform to the target's connectivity constraints?

After this step of the compilation flow, circuits are ready to be executed on the targeted devices (corresponding to the most basic optimization level O0). However, the previous steps significantly increased the size of these circuits-impacting the achievable performance due to the limited coherence time and gate fidelity. Thus, several optimizations may be employed to

\footnotetext{
${ }^{6}$ In fact, the mapping task has been shown to be NP-complete [20].

${ }^{7} \mathrm{~A} S W A P$ operation is eventually realized using three $C N O T$ operations as indicated in the middle of Fig. 1
}

reduce the circuit's size and, hence, improve the actual performance on the quantum computer. Since the IBM Q systems natively support arbitrary single-qubit gates, any number of subsequent single-qubit gates may be fused into one single gate. Additionally, adjacent-gate-cancellations can be used to eliminate instances where a gate is directly followed by its inverse, e.g., two consecutive CNOT operations with the same control and target qubits can be cancelled. These are the most basic optimizations that constitute the standard optimization level O1 of Qiskit. Naturally, more sophisticated optimization techniques have been developed, e.g., gate transformation and commutation [26] (which is included in optimization level O2) or re-synthesis of two-qubit unitary blocks [27] (which is part of the top optimization level O3).

Example 5. Consider again the circuit $\tilde{G}$ from Ex. 4 shown in Fig. Id that has been mapped to the IBM $Q$ London architecture. Applying one-qubit-fusion and adjacent-gate-cancellation eventually allows to eliminate nine single-qubit gates and results in the optimized circuit $G^{\prime}$ shown in Fig. 1e.

\section{B. Verifying Results of the Compilation Flow}

Naturally, it is of utmost importance that the originally intended functionality of a quantum algorithm is preserved throughout the whole compilation flow. This can be guaranteed by verifying the results of the compilation flow. To this end, two possible approaches can be employed: (1) systematically verifying the compilation methods themselves, e.g., by using formal verification techniques [28], [29], or (2) checking the functional equivalence of the original circuit to the respectively compiled circuit [5]-[10]. In this work, we consider the second approach, because, although the first approach allows to guarantee the validity of results for arbitrary circuit inputs, their applicability is severely limited by the effort required to adapt and extend these methods for new developments, such as new optimizations or mapping strategies.

Checking the equivalence between two circuits boils down to checking whether they indeed realize the same functionality. Mathematically, the quantum gates $g_{i}$ of an $n$-qubit quantum circuit $G$ are defined by $2^{n} \times 2^{n}$ unitary matrices $U_{i}$. Consequently, the functionality of a quantum circuit $G$ with gates $g_{0}, \ldots, g_{m-1}$ is described by a unitary matrix $U$, which is obtained by consecutively multiplying the unitary matrix representations $U_{i}$ of each gate $g_{i}$ in reverse order, i.e., $U=U_{m-1} \cdots U_{0}$. Thus, checking the equivalence of two circuits $G$ and $G^{\prime}$ amounts to building and comparing the circuits' system matrices $U$ and $U^{\prime}$. While simple in its concept, the exponential size of the involved matrices quickly renders many direct approaches infeasible 8

In order to cope with this complexity, decision diagrams have been proposed as an efficient data-structure for canoni-

\footnotetext{
${ }^{8}$ Equivalence checking of quantum circuits has even been proven to be QMA-complete in the general case [30].
} 
cally representing and manipulating quantum functionality in the recent past [9], [11], [12] 9 . While decision diagrams indeed frequently allow to represent quantum functionality in a very compact fashion, the decision diagrams corresponding to the circuits $G$ and $G^{\prime}$ may still grow exponentially in the worst case.

Hence, to further improve upon that, a promising approach was recently proposed in [10] utilizing the following observation: Consider two equivalent quantum circuits $G=g_{0} \ldots g_{m-1}$ and $G^{\prime}=g_{0}^{\prime}, \ldots, g_{m^{\prime}-1}^{\prime}$. Then, due to the inherent reversibility of quantum circuits, this certainly allows for the conclusion that $G^{\prime-1} \cdot G=\mathbb{I}$, where $G^{\prime-1}$ denotes the inverse of $G^{\prime}$ and $\mathbb{I}$ denotes the identity function. Moreover, it holds that

$$
\begin{aligned}
\mathbb{I}=G^{\prime-1} \cdot G & =\left(g_{m^{\prime}-1}^{\prime-1} \cdots g_{0}^{\prime-1}\right) \cdot\left(g_{0} \ldots g_{m-1}\right) \\
& \equiv\left(U_{m-1} \cdots U_{0}\right) \cdot\left(U_{0}^{\prime \dagger} \cdots U_{m^{\prime}-1}^{\prime \dagger}\right) \\
& =U_{m-1} \cdots U_{0} \cdot \mathbb{I} \cdot U_{0}^{\prime \dagger} \cdots U_{m^{\prime}-1}^{\prime \dagger} \\
& =: G \rightarrow \mathbb{I} \leftarrow G^{\prime} .
\end{aligned}
$$

As a consequence, checking whether $G$ and $G^{\prime}$ are in fact equivalent can be conducted by starting with the identity and, then, either applying operations of $G$ "from the left" (denoted by $G \rightarrow \mathbb{I}$ ) or (inverted) operations of $G^{\prime}$ "from the right" (denoted by $\mathbb{I} \leftarrow G^{\prime}$ ) until all operations have been applied. If, afterwards, the identity still remains, the circuits $G$ and $G^{\prime}$ have been proven to be equivalent.

The intention of this idea is to keep the intermediate computations as close to the identity as possible, since the identity constitutes the best case for most representations of quantum functionality (e.g., linear in the number of nodes with respect to the number of qubits for decision diagrams).

Example 6. Assume, w.l.o.g, that $m \leq m^{\prime}$, i.e., $G^{\prime}$ has at least as many gates as $G$. Further assume an oracle $\omega: G \rightarrow\left(G^{\prime}\right)^{*}$ exists that, given a gate $g_{i} \in G$, returns a consecutive sequence of gates $g_{k}^{\prime} \ldots g_{l}^{\prime} \in G^{\prime}$ such that $g_{i} \equiv g_{k}^{\prime} \ldots g_{l}^{\prime}$. Then, subsequently applying one gate $g \in G$ and $|\omega(g)|$ inverted gates from $G^{\prime}$ constitutes a "perfect" strategy-yielding the identity after each pair of applications. As a result, only matrices representing, or staying close to, the identity occur. Since these can usually be represented very efficiently using, e.g., decision diagrams, the process of equivalence checking is substantially improved.

However, a major problem remains in how to obtain the "perfect" oracle $\omega(\cdot)$, i.e., in deciding when to apply operations of $G$ ("from the left") and when to apply operations of $G^{\prime}$ ("from the right"). In [10], several strategies for this purpose have been proposed and, indeed, substantial speed-ups in checking the equivalence of two quantum circuits have been achieved with that (cf. Table 1 in [10]). But after all, those

\footnotetext{
${ }^{9}$ Canonicity implies that a comparison of the root pointers of two decision diagrams allows to decide their equivalence.
}

strategies remain rather simple (e.g., they employ a one-to-one or size-proportional application of gates from $G$ and $G^{\prime}$ ) and certainly do not resemble a "perfect" strategy which can indeed keep the computation of $G \rightarrow \mathbb{I} \leftarrow G^{\prime}$ close to the identity.

In contrast, the compilation flow as reviewed in Section III-A provides detailed insights how a circuit $G$ is eventually compiled to a circuit $G^{\prime}$-providing ideal knowledge about how to derive the "perfect" oracle $\omega(\cdot)$. In this work, we propose a verification scheme which uses the idea of applying $G \rightarrow \mathbb{I} \leftarrow G^{\prime}$ and, at the same time, utilizes the knowledge about an actual compilation flow (namely the Qiskit flow). As the experimental evaluations (summarized later in Section V) confirm, this allows for drastic speed-ups and, eventually, makes equivalence checking feasible on a large scale.

\section{Proposed Verification Scheme}

In this section, we propose a verification scheme which rests on the ideas discussed above, but additionally utilizes knowledge about the Qiskit quantum circuit compilation flow to derive a much better oracle $\omega(\cdot)$. For each step in the compilation flow (i.e., for synthesis, mapping, and optimization), a corresponding strategy for $\omega(\cdot)$ is derived which keeps applying $G \rightarrow \mathbb{I} \leftarrow G^{\prime}$ close to the identity. Those strategies are described in the following subsections. Afterwards, they are combined to an overall scheme.

\section{A. Utilizing Knowledge about the Synthesis Step}

Considering the first step of the compilation flow, two issues become relevant for determining the "perfect" $G \rightarrow \mathbb{I} \leftarrow G^{\prime}$ strategy: (1) each gate $g \in G$ is compiled to a sequence of gates $g_{k}^{\prime} \ldots g_{l}^{\prime} \in G^{\prime}$ and (2) the circuits $G$ and $G^{\prime}$ may operate on different numbers of qubits due to the addition of ancillary qubits required for the synthesis.

For the first issue, it can be exploited that the actual decomposition scheme, i.e., into how many elementary gates each of the original circuit's gates is decomposed, is known a priori. Thus, an oracle $\omega(\cdot)$ which, given a gate $g \in G$, returns the corresponding sequence of gates $g_{k}^{\prime} \ldots g_{l}^{\prime} \in G^{\prime}$, is explicitly known in this case. Assuming that $G^{\prime}$ resulted from the synthesis of a given quantum circuit $G$, applying one gate from $G$ and $|\omega(g)|$ inverted gates from $G^{\prime}$ constitutes an optimal strategy for conducting $G \rightarrow \mathbb{I} \leftarrow G^{\prime}$-yielding the identity after each step.

Example 7. Consider the original circuit G shown in Fig. 19 As indicated by Fig. $1 b$ the Toffoli gate of $G$ needs to be decomposed into elementary gates supported by the architecture, while all other gates of $G$ are already supported. Thus, $|\omega(g)|=1$ holds for all $g \in G$ except for the Toffoli gate, where $|\omega(g)|=15$ holds.

In case both circuits do not operate on the same number of qubits, the corresponding unitaries have different dimensions 


$$
U: g_{|1\rangle}^{|0\rangle}\left[\begin{array}{c|c}
U_{00} & U_{10} \\
\hline U_{01} & U_{11}
\end{array}\right]
$$

(a) Original matrix $U$ (b) Modified matrix $\tilde{U}$

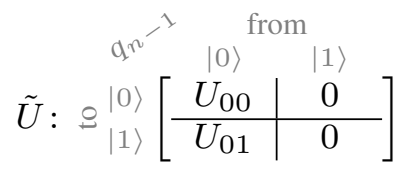

Figure 2: Handling of ancillary qubits

and cannot be applied directly. Unfortunately, it is not sufficient to match the qubit count of $G^{\prime}$ by just augmenting the original circuit with idle qubits. Since ancillary qubits are always initialized in a particular state (typically $|0\rangle$ ), this leaves some degree of freedom in the overall unitary representation $U^{\prime}$. In order to compensate for this degree of freedom, the eventually resulting matrix $U^{\prime}$ has to be modified as shown in the following example.

Example 8. Consider a unitary $2^{n} \times 2^{n}$ matrix $U$ and assume that, w.l.o.g., the last qubit $q_{n-1}$ acts as an ancillary qubit initialized to $|0\rangle$. In general, the action of $U$ depending on the state of $q_{n-1}$ is described by the four $2^{n-1} \times 2^{n-1}$ sub-matrices $U_{i j}$ as illustrated in Fig. $2 a$ Since the ancillary is initialized to $|0\rangle$, the sub-matrices corresponding to the transformation from $|1\rangle$ can be ignored-resulting in the modified matrix $\tilde{U}$ shown in Fig. $2 b$.

\section{B. Utilizing Knowledge about the Mapping Step}

Mapping to the targeted architecture establishes a connection between the circuit's logical and the device's physical qubits. Consequently, while the description of $G$ is expressed in terms of logical qubits $q_{0}, \ldots, q_{n-1}$, the circuit $G^{\prime}$ operates on (a subset of) the device's physical qubits $Q_{0}, \ldots, Q_{N-1}$. If a non-trivial initial mapping (i.e., anything but $Q_{i}: q_{i}$ ) is employed, this leads to the situation that gates from $G^{\prime}$, although functionally equivalent, are applied to different qubits than the gates of $G$. Thus, concluding the equivalence of both circuits is not possible by straight-forwardly using the oracle function $\omega(\cdot)$. Instead, a qubit map $m(\cdot)$ is employed, which stores the mapping between the physical qubits of the circuit $G^{\prime}$ and the logical qubits of the original circuit $G$, i.e., $m\left(Q_{i}\right)=q_{j}$ if physical qubit $Q_{i}$ is initially assigned logical qubit $q_{j}$. Whenever a gate from $G^{\prime}$ is to be applied to a certain physical qubit $Q_{i}$, this is translated to the corresponding logical qubit $m\left(Q_{i}\right)=q_{j}$-again allowing to stay close to the identity.

Example 9. Consider the original circuit $G$ and the mapped circuit $\tilde{G}$ shown in Fig. 1 a and Fig. $1 d$ respectively. While the $X$ gate at the beginning of $G$ is applied to the logical qubit $q_{2}$, it is applied to the physical qubit $Q_{1}$ in the circuit $\tilde{G}$. In order to fix this mismatch, the qubit map $m(\cdot)$-mapping $Q_{0} \mapsto q_{0}$, $Q_{1} \mapsto q_{2}$, and $Q_{2} \mapsto q_{1}$-is employed. Consequently, the $X$ gate of $\tilde{G}$ is applied to $m\left(Q_{1}\right)=q_{2}$ which now matches the original gate from $G$ perfectly.

However, as discussed in Section [III-A the logical-to-physical qubit mapping of a compiled circuit in general changes dynamically throughout the circuit in order to satisfy all constraints imposed by the device's coupling map. As a consequence, the potential of using the (static) qubit map $m(\cdot)$ in combination with the oracle function $\omega(\cdot)$ to stay close to the identity is significantly diminished. That is, because the dynamically changed mapping again results in a scenario where gates from $G^{\prime}$ are applied to different qubits than in the circuit $G$. Therefore, a perfect verification strategy needs to keep track of the changes in the logical-to-physical qubit mapping caused by $S W A P$ operation 10 and, accordingly, needs to update the qubit map $m(\cdot)$ throughout the verification procedure.

Example 10. Consider again the scenario of Ex. 9. If the $G \rightarrow \mathbb{I} \leftarrow G^{\prime}$ scheme is carried out using the qubit map $m(\cdot)$ defined there, the result would not represent the identity. That is, because the logical-to-physical qubit mapping is changed in the middle of $\tilde{G}$ by a SWAP operation applied to $Q_{0}$ and $Q_{1}$. Thus, at that specific point, the qubit map $m(\cdot)$ has to be updated accordingly, i.e., it then has to map $Q_{0} \mapsto q_{2}$, $Q_{1} \mapsto q_{0}$, and $Q_{2} \mapsto q_{1}$. Through this dynamic change, the computation of $G \rightarrow \mathbb{I} \leftarrow G^{\prime}$ remains close to the identity and, eventually, proves the equivalence of both circuits.

\section{Utilizing Knowledge about the Optimization Step}

If no optimizations were to be applied to the circuit resulting from the synthesis and mapping step (which is equivalent to applying Qiskit's O0 optimization level), the strategies proposed above allow to conduct $G \rightarrow \mathbb{I} \leftarrow G^{\prime}$ in a perfect fashion-yielding the identity after each step. However, optimizations as discussed in Section III-A further alter the circuit-making it harder to verify the resulting circuit. In the following, we cover how to anticipate the effects of the two most common optimizations employed in Qiskit—single-qubit gate fusion and adjacent gate cancellation (see Section [III-A).

Example 11. Consider again the circuit $\tilde{G}$ shown in Fig. $1 d$. There, the grey box indicates the gates of $\tilde{G}$ realizing the Toffoli gate of the original circuit $G$ shown in Fig. 1a. The middle qubit thereby contains a $T$ gate, which is directly followed by an $H$ gate. Accordingly, in the optimized circuit shown in Fig. 1e. these have been merged into a single $U_{2}\left(0, \frac{5 \pi}{4}\right)$ gate. Thus, $|\omega(g)|=15$ does no longer hold, but has to be modified to $|\omega(g)|=14$ instead in case of the Toffoli gate (see Ex. 7).

In addition to anticipating fusions within individual gate realizations through adaptations of the oracle function $\omega(\cdot)$, a pre-processing pass is conducted which fuses consecutive

\footnotetext{
${ }^{10} S W A P$ s can be reconstructed from consecutive sequences of three
} CNOTs in $G^{\prime}$ as indicated in the middle of Fig. 1 


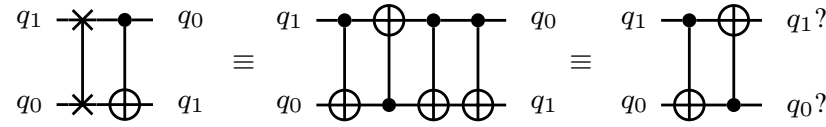

Figure 3: $S W A P$ and $C N O T$ cancellation

single-qubit gates where they are present in the original circuit $G$ (e.g., fusing the $H-X-H$ cascade at the end of the circuit $G$ shown in Fig. 1 a to a single $Z$ gate). However, reductions across multiple gates that were decomposed during synthesis cannot be accounted for in this fashion. Thus, the formerly constructed perfect oracle function $\omega(\cdot)$ becomes approximate.

Example 12. Consider again the circuit $\tilde{G}$ shown in Fig. $1 d$ and its optimized variant $G^{\prime}$ shown in Fig. 1e. Then, the cancellation of the two consecutive $H$ gates in the beginning of $\tilde{G}$ cannot be anticipated through a straightforward adaptation of $\omega(\cdot)$. However, as also confirmed by the experimental evaluations in Section $V, \omega(\cdot)$ remains a suitable approximation for staying close to the identity.

The second optimization employed per default-adjacent gate cancellation-introduces a peculiar issue for the verification strategy as shown in the following example.

Example 13. Consider a SWAP operation directly followed by a CNOT operation. Since the SWAP operation itself is realized by three consecutive CNOTs, this sequence of operations may be simplified by cancelling two of them as shown in Fig. 3 While the qubit map $m(\cdot)$ can be easily adapted in the first two cases, the optimized circuit shows no sign of an applied SWAP and, furthermore, introduces an additional CNOT gate to the compiled circuit which previously did not exist in $G$. This makes it hard for the proposed strategies to still identify the SWAP and, hence, update the qubit map $m(\cdot)$ as described in the previous section.

As a solution, any occurrence of two consecutive CNOT operations in $G^{\prime}$ as shown on the right of Fig. 3 that is not followed by a third matching CNOT is substituted by the sequence shown on the left of Fig. 3. Overall, this again allows to accurately track the qubit mapping $m(\cdot)$ and conduct the equivalence check in an optimal fashion.

\section{Resulting Verification Scheme}

All of the considerations above finally result in a dedicated verification scheme that is tailored for verifying results of the Qiskit compilation flow. First of all, a pre-processing step fuses subsequent single-qubit gates in the circuit $G$ and substitutes $S W A P$ (and possibly a CNOT) gates where applicable in the circuit $G^{\prime}$. Afterwards, if necessary, the circuit $G$ is augmented with idle ancillary qubits. Then, the general $G \rightarrow \mathbb{I} \leftarrow G^{\prime}$ scheme is employed-utilizing the oracle function $\omega(\cdot)$ to determine which gates from $G^{\prime}$ are to be applied for each application of a gate from $G$.

The actual application of gates from $G^{\prime}$ happens with regard to the qubit map $m(\cdot)$ which establishes the connection between the circuit $G$ 's logical qubits and $G^{\prime}$ 's physical qubits. During these steps, this qubit map is dynamically updated to account for the insertion of $S W A P$ operations during the mapping. After applying all gates from both circuits, the result of this scheme is modified as illustrated in Ex. 8. Eventually, the two circuits are shown to be equivalent if the modified result resembles the identity for the non-ancillary qubits.

As confirmed by the experimental evaluations, which are summarized next, this scheme allows to efficiently verify even large instances consisting of tens of thousands of gates within seconds. Additionally, in contrast to formally verifying the individual compilation steps (see Section III-B), this approach remains generic enough to work well out of the box, even when optimizations are employed that have not been directly accounted for, e.g., commutation rules.

\section{EXPERIMENTAL EVALUATIONS}

The proposed verification scheme has been implemented on top of the tool proposed in [10] (which is available at https://iic.jku.at/eda/research/quantum_verification). More precisely, we took the recent version (revision 1.2) and extended this tool with the strategies described in Section IV Afterwards, we conducted extensive experiments to evaluate the performance of the resulting approach. In this section, we summarize our evaluations. To this end, we first briefly review the setup and, afterwards, present as well as discuss the obtained results.

\section{A. Setup}

In our evaluations, we considered circuits that are frequently used to benchmark compilers. Using IBM Qiskit [1] (specifically, Qiskit Terra 0.12.0), each original circuit $G$ has been compiled for a specific target device-resulting in an alternative circuit $G^{\prime}$. During this process, multi-controlled Toffoli gates have been synthesized using the "basic" mode (yielding the smallest circuits, with the highest number of additionally needed qubits) and the target device has been chosen as the smallest possible one capable of accommodating the resulting number of qubits. Specifically, IBM Q Boeblingen has been chosen for circuits with up to 20 qubits, while $I B M$ $Q$ Rochester has been used for circuits with up to 53 qubits.

As discussed in Section III-A. Qiskit offers several optimization levels for compiling circuits. In our evaluations, we considered Qiskit's default optimization level (i.e., O1) and-in order to show the proposed approach's applicability to scenarios it has not been explicitly tailored towards-the more advanced $\mathrm{O} 2$ level. All evaluations have been performed on a $4 \mathrm{GHz}$ Amazon EC2 zld instance running Ubuntu 18.04 
with at least $32 \mathrm{~GB}$ per job using GNU Parallel [31]. A hard timeout of $1 \mathrm{~h}$ (i.e., $3600 \mathrm{~s}$ ) was set for each run.

\section{B. Obtained Results}

In a first series of evaluations, we considered Qiskit's default optimization level O1. A subset of the respectively obtained results is shown in Table $\|^{1}$. Here, the first columns describe the original circuit $G$ (its name, number of qubits, and number of gates) as well as the device the circuit was mapped to. Then, the size of the resulting circuit $G^{\prime}$ is listed, along with the runtimes of (1) the equivalence checking routine from [11], (2) its advanced improvement from [10] (utilizing $G \rightarrow \mathbb{I} \leftarrow$ $G^{\prime}{ }^{12}$ and (3) the strategy proposed in this work.

The results clearly show the superiority of the proposed method. While the first approach using $G \rightarrow \mathbb{I} \leftarrow G^{\prime}$ (as proposed in [10]) allows to reduce the equivalence checking runtime down to a third or a half in most cases (sometimes even more), substantial runtimes (or even timeouts) are still reported. On the contrary, additionally exploiting explicit knowledge about the compilation flow as proposed in this work allows for drastic further improvements. In fact, all considered instances are successfully verified within (fractions of) seconds, whereas state-of-the-art equivalence checking methods and even the recently proposed advanced techniques frequently time-out or require substantial runtime.

In a second series of evaluations, we considered Qiskit's more advanced optimization level O2. In this regard, Table II shows a representative subset of the obtained result ${ }^{13}$. While the proposed methodology was explicitly tailored for the default optimization level of Qiskit, i.e., O1, its performance remains almost on an equally high level in case of verifying circuits compiled with optimization level $\mathrm{O} 2$-where several more advanced optimization techniques, such as gate commutation rules, are employed, which are not directly accounted for in the proposed scheme. This shows that even utilization of partial knowledge about the underlying compilation flow is sufficient to drastically improve the verification of the correctness of its result.

\section{CONCLUSION}

In this work, we proposed a dedicated scheme for verifying results of the IBM Qiskit compilation flow. To this end, we exploit characteristics unique to quantum computing and explicitly incorporate knowledge about the compilation flow in order to design a strategy that allows to keep the overhead

\footnotetext{
${ }^{11}$ Due to space limitations, only a small subset of benchmarks is listed here. However, the proposed scheme is publicly available at https://github. com/iic-jku/qcec to conduct further evaluations.

${ }^{12}$ The "Proportional" strategy was used, as it produced the best results in [10]

${ }^{13}$ In some instances, the circuit resulting from optimization level $\mathrm{O} 2$ is actually larger than the circuit resulting from O1. This is due to the fact that the default $S W A P$-insertion technique employed in Qiskit is stochastic. However, this does not influence the general observations gained from this evaluation, since the actual number of $S W A P$ s actually does not influence the performance of the proposed strategy.
}

of verifying compilation results minimal. Experimental evaluations confirm that the proposed strategy consistently allows to verify instances with more than ten-thousand gates within seconds-even if optimizations are employed which are not directly accounted for. Compared to the state of the art, which often requires substantial runtimes or even time-outs in these tasks, this is a drastic improvement. The resulting tool is publicly available at https://github.com/iic-jku/qcec and can easily be adapted to different compilation flows or additional optimizations in the future.

\section{ACKNOWLEDGMENTS}

This work has partially been supported by the LIT Secure and Correct Systems Lab funded by the State of Upper Austria as well as by the BMK, BMDW, and the State of Upper Austria in the frame of the COMET program (managed by the FFG).

\section{REFERENCES}

[1] G. Aleksandrowicz et al., "Qiskit: An open-source framework for quantum computing", 2019.

[2] Cirq: A python framework for creating, editing, and invoking Noisy Intermediate Scale Quantum (NISQ) circuits. [Online]. Available: https://github.com/quantumlib/Cirq (visited on 04/10/2020).

[3] Quantum Development Kit, Microsoft. [Online]. Available: https://microsoft.com/en-us/quantum/development-kit (visited on $03 / 25 / 2020$ ).

[4] Forest SDK, Rigetti. [Online]. Available: https://rigetti.com/ forest (visited on 04/10/2020).

[5] G. F. Viamontes, I. L. Markov, and J. P. Hayes, "Checking equivalence of quantum circuits and states", in Int'l Conf. on $C A D, 2007$.

[6] S. Yamashita and I. L. Markov, "Fast equivalence-checking for quantum circuits", in Int'l Symp. on Nanoscale Architectures, 2010.

[7] K. N. Smith and M. A. Thornton, "A quantum computational compiler and design tool for technology-specific targets", in Int'l Symp. on Computer Architecture, 2019, pp. 579-588.

[8] P. Niemann, R. Wille, and R. Drechsler, "Equivalence checking in multi-level quantum systems", in Int'l Conf. of Reversible Computation, 2014.

[9] S.-A. Wang, C.-Y. Lu, I.-M. Tsai, and S.-Y. Kuo, "An XQDDbased verification method for quantum circuits", in IEICE Trans. Fundamentals, 2008, pp. 584-594.

[10] L. Burgholzer and R. Wille, "Advanced equivalence checking of quantum circuits", ArXiv: 2004.08420 [quant-ph], 2020.

[11] P. Niemann, R. Wille, D. M. Miller, M. A. Thornton, and R. Drechsler, "QMDDs: Efficient quantum function representation and manipulation", IEEE Trans. on CAD of Integrated Circuits and Systems, vol. 35, no. 1, pp. 86-99, 2016.

[12] A. Zulehner, S. Hillmich, and R. Wille, "How to efficiently handle complex values? Implementing decision diagrams for quantum computing", in Int'l Conf. on CAD, 2019.

[13] M. A. Nielsen and I. L. Chuang, Quantum Computation and Quantum Information. Cambridge University Press, 2010.

[14] IBM Q, IBM Q. [Online]. Available: https://www.research. ibm.com/ibm-q/ (visited on 04/10/2020). 
Table I: Optimization Level O1

\begin{tabular}{|c|c|c|c|c|c|c|c|}
\hline \multicolumn{4}{|c|}{ Benchmark } & \multicolumn{4}{|c|}{ Results } \\
\hline Name & $n$ & $|G|$ & Architecture & $\left|G^{\prime}\right|$ & $t_{\text {sota }}[\mathrm{s}]$ & $t_{a d v}[\mathrm{~s}]$ & $t_{\text {prop }}[\mathrm{s}]$ \\
\hline sym9_317 & 27 & 64 & Rochester & 1540 & $>3600.00$ & 63.61 & 0.03 \\
\hline c2_182 & 35 & 305 & Rochester & 2545 & $>3600.00$ & 2500.79 & 0.10 \\
\hline cm163a_213 & 29 & 39 & Rochester & 3539 & $>3600.00$ & 183.93 & 0.02 \\
\hline apla_203 & 22 & 80 & Rochester & 13548 & $>3600.00$ & $>3600.00$ & 0.10 \\
\hline cm151a_211 & 28 & 33 & Rochester & 3889 & $>3600.00$ & $>3600.00$ & 0.03 \\
\hline rd84_313 & 34 & 113 & Rochester & 2295 & $>3600.00$ & $>3600.00$ & 2.64 \\
\hline mod5adder_306 & 32 & 110 & Rochester & 2348 & $>3600.00$ & $>3600.00$ & 0.22 \\
\hline add6_196 & 19 & 229 & Rochester & 25460 & 1513.95 & 514.19 & 0.51 \\
\hline cycle177_3_112 & 20 & 48 & Rochester & 14987 & 1111.38 & 1284.86 & 0.34 \\
\hline rd73_312 & 25 & 76 & Rochester & 1426 & 1108.62 & 196.10 & 0.20 \\
\hline cm150a_210 & 22 & 53 & Rochester & 3788 & 761.82 & 577.95 & 0.05 \\
\hline ryy6_256 & 17 & 44 & Rochester & 13103 & 504.42 & 316.13 & 0.08 \\
\hline c2_181 & 35 & 116 & Rochester & 2708 & 403.80 & 4.06 & 0.03 \\
\hline alu3_200 & 18 & 94 & Rochester & 10285 & 255.25 & 151.17 & 0.07 \\
\hline decod_217 & 21 & 80 & Rochester & 6197 & 192.30 & 121.17 & 0.05 \\
\hline example2_231 & 16 & 157 & Rochester & 19411 & 187.63 & 79.62 & 0.21 \\
\hline cu_219 & 25 & 40 & Rochester & 5031 & 177.15 & 72.20 & 0.02 \\
\hline plus63mod8192_164 & 13 & 492 & Rochester & 111720 & 166.02 & 112.40 & 1.85 \\
\hline C7552_205 & 21 & 80 & Rochester & 6384 & 158.30 & 80.38 & 0.05 \\
\hline alu2_199 & 16 & 157 & Rochester & 19007 & 139.00 & 68.86 & 0.17 \\
\hline dk17_224 & 21 & 49 & Rochester & 6650 & 105.19 & 36.56 & 0.04 \\
\hline mlp4_245 & 16 & 131 & Rochester & 14513 & 79.06 & 22.69 & 0.09 \\
\hline mux_246 & 22 & 35 & Rochester & 3679 & 71.17 & 39.75 & 0.04 \\
\hline urf1_150 & 9 & 1517 & Boeblingen & 134216 & 68.45 & 43.96 & 9.52 \\
\hline clip_206 & 14 & 174 & Rochester & 20872 & 62.91 & 43.62 & 0.20 \\
\hline plus63mod4096_163 & 12 & 429 & Rochester & 82195 & 58.47 & 44.01 & 1.46 \\
\hline dist_223 & 13 & 185 & Boeblingen & 19458 & 19.93 & 12.08 & 0.13 \\
\hline inc_237 & 16 & 93 & Rochester & 7523 & 18.02 & 8.46 & 0.04 \\
\hline urf5_159 & 9 & 499 & Boeblingen & 57099 & 15.99 & 9.17 & 0.90 \\
\hline alu1_198 & 20 & 32 & Rochester & 1377 & 13.19 & 4.02 & 0.01 \\
\hline cmb_214 & 20 & 18 & Rochester & 2589 & 11.47 & 10.76 & 0.01 \\
\hline sqr6_259 & 18 & 81 & Rochester & 4436 & 10.89 & 4.50 & 0.02 \\
\hline 5xp1_194 & 17 & 85 & Rochester & 5487 & 10.78 & 4.59 & 0.04 \\
\hline rd84_253 & 12 & 111 & Boeblingen & 7857 & 9.99 & 6.78 & 0.05 \\
\hline root_255 & 13 & 99 & Boeblingen & 8594 & 7.97 & 5.15 & 0.05 \\
\hline
\end{tabular}

$n$ : Number of qubits $|G|$ : Gate count of $G \quad$ Architecture: Boeblingen (20 qubits) or Rochester (53 qubits) $\left|G^{\prime}\right|$ : Gate count of $G^{\prime} \quad t_{\text {sota }}$ : Runtime of state-of-the-art EC routine [11]

$t_{a d v}$ : Runtime of advanced methodology EC routine [10] $\quad t_{\text {prop }}$ : Runtime of proposed, dedicated EC scheme

[15] L. K. Grover, "A fast quantum mechanical algorithm for database search", Proc. of the ACM, pp. 212-219, 1996.

[16] A. Barenco et al., "Elementary gates for quantum computation", Phys. Rev. A, vol. 52, no. 5, pp. 3457-3467, 1995.

[17] D. Maslov, "On the advantages of using relative phase Toffolis with an application to multiple control Toffoli optimization", Phys. Rev. A, vol. 93, no. 2, p. 022 311, 2016.

[18] R. Wille, M. Soeken, C. Otterstedt, and R. Drechsler, "Improving the mapping of reversible circuits to quantum circuits using multiple target lines", in Asia and South Pacific Design Automation Conf., 2013.

[19] P. Murali, J. M. Baker, A. Javadi-Abhari, F. T. Chong, and M. Martonosi, "Noise-adaptive compiler mappings for Noisy Intermediate-Scale Quantum computers", in Int'l Conf. on Architectural Support for Programming Languages and $\mathrm{Op}$ erating Systems, 2019, pp. 1015-1029.

[20] M. Y. Siraichi, V. F. dos Santos, S. Collange, and F. M. Q. Pereira, "Qubit allocation", in Proc. Int'l Symp. on Code Generation and Optimization, 2018, pp. 113-125.
[21] A. Zulehner, A. Paler, and R. Wille, "An efficient methodology for mapping quantum circuits to the IBM QX architectures", IEEE Trans. on CAD of Integrated Circuits and Systems, vol. 38, no. 7, pp. 1226-1236, 2019.

[22] R. Wille, L. Burgholzer, and A. Zulehner, "Mapping quantum circuits to IBM QX architectures using the minimal number of SWAP and H operations", in Design Automation Conf., 2019.

[23] G. Li, Y. Ding, and Y. Xie, "Tackling the qubit mapping problem for NISQ-era quantum devices", in Int'l Conf. on Architectural Support for Programming Languages and Operating Systems, 2019.

[24] A. Matsuo, W. Hattori, and S. Yamashita, "Reducing the overhead of mapping quantum circuits to IBM Q system", in IEEE International Symposium on Circuits and Systems, 2019.

[25] M. Amy and V. Gheorghiu, "Staq - A full-stack quantum processing toolkit", ArXiv:1912.06070, 2019.

[26] T. Itoko, R. Raymond, T. Imamichi, and A. Matsuo, "Optimization of quantum circuit mapping using gate transformation and commutation", Integration, vol. 70, pp. 43-50, 2020. 
Table II: Optimization Level O2

\begin{tabular}{|c|c|c|c|c|c|c|c|}
\hline \multicolumn{4}{|c|}{ Benchmark } & \multicolumn{4}{|c|}{ Results } \\
\hline Name & $n$ & $|G|$ & Architecture & $\left|G^{\prime}\right|$ & $t_{\text {sota }}[\mathrm{s}]$ & $t_{a d v}[\mathrm{~s}]$ & $t_{\text {prop }}[\mathrm{s}]$ \\
\hline sym9_317 & 27 & 64 & Rochester & 1566 & $>3600.00$ & 81.40 & 0.03 \\
\hline c2_182 & 35 & 305 & Rochester & 2692 & 963.18 & 99.00 & 0.22 \\
\hline cm163a_213 & 29 & 39 & Rochester & 3704 & 2004.05 & 515.69 & 0.03 \\
\hline apla_20 & 22 & 80 & Rochester & 13212 & $>3600.00$ & $>3600.00$ & 0.11 \\
\hline cm151a_211 & 28 & 33 & Rochester & 3798 & $>3600.00$ & $>3600.00$ & 0.04 \\
\hline rd84_313 & 34 & 113 & Rochester & 2158 & $>3600.00$ & $>3600.00$ & 12.47 \\
\hline mod5adder_306 & 32 & 110 & Rochester & 2380 & $>3600.00$ & $>3600.00$ & 0.37 \\
\hline add6_196 & 19 & 229 & Rochester & 26201 & 455.70 & 138.65 & 1.29 \\
\hline cycle17_3_112 & 20 & 48 & Rochester & 13876 & 777.82 & 667.16 & 1.80 \\
\hline rd73_312 & 25 & 76 & Rochester & 1498 & 199.84 & 226.25 & 0.65 \\
\hline cm150a_210 & 22 & 53 & Rochester & 3678 & 795.15 & 583.42 & 0.07 \\
\hline ryy6_256 & 17 & 44 & Rochester & 13100 & 22.17 & 23.40 & 0.20 \\
\hline c2_181 & 35 & 116 & Rochester & 3271 & $>3600.00$ & $>3600.00$ & 0.04 \\
\hline alu3_200 & 18 & 94 & Rochester & 10463 & 44.81 & 27.40 & 0.11 \\
\hline decod_217 & 21 & 80 & Rochester & 6289 & 35.55 & 23.60 & 0.05 \\
\hline example2_231 & 16 & 157 & Rochester & 19207 & 38.57 & 16.77 & 0.62 \\
\hline cu_219 & 25 & 40 & Rochester & 4863 & 136.76 & 48.83 & 0.03 \\
\hline plus63mod8192_164 & 13 & 492 & Rochester & 111258 & 110.21 & 78.31 & 8.61 \\
\hline C7552_205 & 21 & 80 & Rochester & 6291 & 60.18 & 32.07 & 0.04 \\
\hline alu2_199 & 16 & 157 & Rochester & 19080 & 23.56 & 12.42 & 0.27 \\
\hline dk17_224 & 21 & 49 & Rochester & 6589 & 7.70 & 2.99 & 0.05 \\
\hline mlp4_245 & 16 & 131 & Rochester & 14394 & 12.41 & 4.59 & 0.17 \\
\hline mux_246 & 22 & 35 & Rochester & 3829 & 156.01 & 91.49 & 0.06 \\
\hline urf1_150 & 9 & 1517 & Boeblingen & 132898 & 75.19 & 44.03 & 21.89 \\
\hline clip_206 & 14 & 174 & Rochester & 21407 & 44.16 & 23.82 & 0.26 \\
\hline plus63mod4096_163 & 12 & 429 & Rochester & 83835 & 67.01 & 45.24 & 4.71 \\
\hline dist_223 & 13 & 185 & Boeblingen & 18721 & 28.09 & 16.70 & 0.24 \\
\hline inc_237 & 16 & 93 & Rochester & 7624 & 55.03 & 42.83 & 0.05 \\
\hline urf5_159 & 9 & 499 & Boeblingen & 56089 & 15.52 & 8.77 & 3.00 \\
\hline alu1_198 & 20 & 32 & Rochester & 1424 & 14.56 & 1.42 & 0.01 \\
\hline cmb_214 & 20 & 18 & Rochester & 2669 & 15.03 & 15.31 & 0.02 \\
\hline sqr6_259 & 18 & 81 & Rochester & 4536 & 5.31 & 2.03 & 0.03 \\
\hline 5xp1_194 & 17 & 85 & Rochester & 5420 & 6.89 & 3.52 & 0.04 \\
\hline rd84_253 & 12 & 111 & Boeblingen & 7715 & 4.48 & 2.77 & 0.06 \\
\hline root_255 & 13 & 99 & Boeblingen & 8729 & 6.10 & 3.82 & 0.06 \\
\hline
\end{tabular}

$n$ : Number of qubits $|G|$ : Gate count of $G \quad$ Architecture: Boeblingen (20 qubits) or Rochester (53 qubits) $\left|G^{\prime}\right|$ : Gate count of $G^{\prime} \quad t_{\text {sota }}$ : Runtime of state-of-the-art EC routine [11]

$t_{a d v}$ : Runtime of advanced methodology EC routine [10] $t_{\text {prop }}$ : Runtime of proposed, dedicated EC scheme

[28] K. Hietala, R. Rand, S.-H. Hung, X. Wu, and M. Hicks, "A verified optimizer for quantum circuits", ArXiv:1912.02250, 2019.

[29] Y. Shi et al., "Contract-based verification of a realistic quantum compiler", ArXiv:1908.08963, 2019.

[30] D. Janzing, P. Wocjan, and T. Beth, ""Non-identity check" is QMA-complete", Int. J. Quantum Inform., vol. 03, no. 03, pp. 463-473, 2005.
[27] G. Vidal and C. M. Dawson, "Universal quantum circuit for two-qubit transformations with three controlled-NOT gates", Phys. Rev. A, vol. 69, no. 1, p. $010301,2004$.

[31] O. Tange, GNU Parallel 2018. Ole Tange, 2018. 